2. A. H. Copeland, Admissible numbers in the theory of probability, Amer. J. Math. vol. 50 (1928) pp. 535-552.

3. F. Hausdorff, Mengenlehre, 3rd ed., Berlin, 1935.

4. B. Rankin, Computable probability spaces, Acta Math. vol. 103 (1960) pp. 89122.

5. H. Reichenbach, The theory of probability, Berkeley, 1949.

6. M. Sekanina, On a certain characteristic of compact connected sets in euclidean space, Casopia Pěst. Mat. vol. 82 (1957) pp. 129-136 (Czech) (reviewed in Math. Reviews vol. 19 (1958) p. 667).

7. J. Ville, Étude critique de la notion de collectif, Paris, 1939.

Case Institute of Technology

\title{
EXACT SEPARATION OF RECURSIVELY ENUMERABLE SETS WITHIN THEORIES
}

HILARY PUTNAM AND RAYMOND M. SMULLYAN

Introduction. Ehrenfeucht and Feferman [1] have recently shown that every r.e. (recursively enumerable) set is representable in every consistent axiomatizable extension $(T)$ of Robinson's system $R$ (see $[2$, p. 53]). In this paper we extend the above result and show that any such theory $(T)$ has the stronger property that for any two disjoint r.e. sets $A, B$ there is a formula $F(x)$ which represents $A$ and whose negation $F^{\prime}(x)$ (i.e. $\sim F(x)$ ) represents $B$.

The proof of Ehrenfeucht and Feferman uses Myhill's result [3] that every creative set is universal. Our proof analogously uses the recent result first proved by Muchnik [4], and independently by Smullyan [5] - that every effectively inseparable pair of r.e. sets is doubly universal (cf. Theorem A, §3).

1. Terminology. We let $(T)$ be any theory in standard formalization (cf. Tarski [2]). For any number $n$ we let $\bar{n}$ (written " $\Delta_{n}$ " in Tarski) be the numeral associated with $n$. For any formula $F(x)$ we let $F_{T}$ be the set of all numbers $n$ such that $F(\bar{n})$ is provable in $(T)$ and we let $F_{R}$ be the set of all $n$ such that $F(\bar{n})$ is refutable in $(T)$-i.e. such that $F^{\prime}(\bar{n})$ is provable in $(T)$. (For a consistent theory $(T)$, the sets $F_{T}, F_{R}$ are, of course, disjoint.) Let $A, B$ be disjoint number sets. We say that $F$ (i.e. $F(x)$ ) represents $A$ iff $A=F_{T}$. We say that $F$ separates $A$ from $B$ within $(T)$, or that $F$ separates the pair $(A, B)$ in $(T)$, iff $A \subseteq F_{T}$ and $B \subseteq F_{R}$. (This means that $F, F^{\prime}$ respectively represent supersets of $A, B$.) And we say that $F$ exactly separates the pair $(A, B)$

Presented to the Society, April 25, 1959 under the title Double isomorphism of Rosser theories; received by the editors October 5, 1959. 
within (T) iff $F$ represents $A$ and $F^{\prime}$ represents $B$-i.e. iff $A=F_{T}$ and $B=F_{R}$. In [5] $(T)$ is called a Rosser theory iff every disjoint pair of r.e. sets is separable in $(T)$, and an exact Rosser theory iff every such pair is exactly separable in $(T)$. It is obvious that every extension of a Rosser theory is again a Rosser theory.

2. A preliminary lemma. A function $f(x)$ is called definable in $(T)$ iff there is a formula $F(x, y)$ such that for every pair of numbers $m, n$ the following conditions hold:

(i) If $f(m)=n$ then $\vdash F(\bar{m}, \bar{n})$,

(ii) If $f(m) \neq n$ then $\vdash F^{\prime}(\bar{m}, \bar{n})$,

(iii) If $f(m)=n$ then $\vdash(\forall y)(F(\bar{m}, y) \supset y=\bar{n})$.

LEMMA 1. Let $(T)$ be a consistent theory in which $f(x)$ is definable and in which $A$ is exactly separable from $B$. Then $f^{-1}(A)$ is exactly separable from $f^{-1}(B)$ within $(T) .^{2}$

Proof. Let $F(x, y)$ be a formula which defines $f(x)$; let $G(x)$ exactly separate $(A, B)$ in $(T)$; let $H(x)$ be the formula $(\exists y)(F(x, y) \wedge G(y))$. We show that $H$ exactly separates $f^{-1}(A)$ from $f^{-1}(B)$ within $(T)$.

Let $n$ be any number and let $m=f(n)$.

(i) Suppose $n \in f^{-1}(A)$. Then $m \in A$. Then $\vdash G(\bar{m})$ (since $G$ represents $A$ ). Also $\vdash F(\bar{n}, \bar{m})$ (since $F$ defines $f$ ). Hence $\vdash F(\bar{n}, \bar{m}) \wedge G(\bar{m})$. Hence $\vdash(\exists y)(F(\bar{n}, y) \wedge G(y))$-i.e. $\vdash H(\bar{n})$.

(ii) Conversely, suppose $\vdash H(\bar{n})$-i.e. $\vdash(\exists y)(F(\bar{n}, y) \wedge G(y))$. Since $F$ defines $f$ then $\vdash F(\bar{n}, y) \supset y=\bar{m}$. Hence $\vdash(F(\bar{n}, y) \wedge G(y))$ $\supset(y=\bar{m} \wedge G(y))$. And since $\vdash(\exists y)(F(\bar{n}, y) \wedge G(y))$, we have $\vdash(\exists y)(y=\bar{m} \wedge G(y))$. Hence $\vdash G(\bar{m})$. Then $m \in A$. So $n \in f^{-1}(A)$.

By (i) and (ii), $H$ represents $f^{-1}(A)$.

(iii) Suppose $n \in f^{-1}(B)$. Then $m \in B$. Then $\vdash G^{\prime}(\bar{m})$. Then $\vdash y$ $=\bar{m} \supset G^{\prime}(y)$. And since $\vdash F(\bar{n}, y) \supset y=\bar{m}$, we have $\vdash F(\bar{n}, y) \supset G^{\prime}(y)$. Hence $\vdash \sim(F(\bar{n}, y) \wedge G(y))$; hence $\vdash(\forall y) \sim(F(\bar{n}, y) \wedge G(y))$. Thus $\vdash \sim(\exists y)(F(\bar{n}, y) \wedge G(y))$-i.e. $\vdash H^{\prime}(\bar{n})$.

(iv) Conversely, suppose $\vdash H^{\prime}(\bar{n})$-i.e. $\vdash \sim(\exists y)(F(\bar{n}, y) \wedge G(y))$. Then $\vdash(\forall y)\left(F(\bar{n}, y) \supset G^{\prime}(y)\right)$, and so $\vdash F(\bar{n}, \bar{m}) \supset G^{\prime}(\bar{m})$. Also $\vdash F(\bar{n}, \bar{m})$. Hence $\vdash G^{\prime}(\bar{m})$, so $m \in B$ and $n \in f^{-1}(B)$.

By (iii) and (iv), $H^{\prime}$ represents $B$. This completes the proof.

3. Doubly universal sets. A pair $\left(U_{1}, U_{2}\right)$ of number sets is called doubly universal iff for every disjoint pair $(A, B)$ of r.e. sets, there is a 1-1 recursive function $f(x)$ such that $A=f^{-1}\left(U_{1}\right)$ and $B=f^{-1}\left(U_{2}\right)$.

${ }^{1}$ For any formula $X$, we use the notation " $-X$ " to mean that $X$ is provable (valid) in $(T)$.

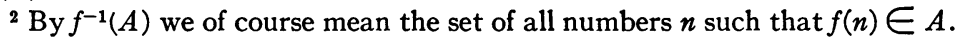


(This means that for every number $n: n \in A \equiv f(n) \in U_{1}$ and $n \in B$ $\equiv f(n) \in U_{2}$.)

From Lemma 1 immediately follows:

THEOREM 1. If $(T)$ is a consistent theory in which every recursive function ${ }^{3}$ is definable and in which at least one doubly universal pair is exactly separable, $(T)$ is an exact Rosser theory (i.e. every disjoint pair of r.e. sets is then exactly separable in $(T)$ ).

4. Effectively inseparable sets. We consider the Post-Kleene enumeration $\omega_{1}, \omega_{2}, \cdots, \omega_{i}, \cdots$ of all r.e. sets. ${ }^{4} \mathrm{~A}$ disjoint pair $(A, B)$ of number sets is called effectively inseparable (henceforth abbreviated "E.I.") iff there is a recursive function $\delta(x, y)$ such that for any numbers $i, j$ such that $\omega_{i}, \omega_{j}$ are disjoint supersets of $A, B$ respectively, the number $\delta(i, j)$ is outside both $\omega_{i}$ and $\omega_{j}$.

The following theorem was proved in [4] and [5] (cf. introduction).

THEOREM A. Every effectively inseparable pair of r.e. sets is doubly universal.

From Theorem 1 and Theorem A immediately follows:

THEOREM 2. If $(T)$ is a consistent theory in which every recursive function is definable and in which some E.I. pair of r.e. sets is exactly separable, then $(T)$ is an exact Rosser theory.

We now need:

Lemma 2. If some E.I. pair of sets is separable in $(T)$ and if $(T)$ is axiomatizable then some E.I. pair of r.e. sets is exactly separable in $(T)$.

Proof. Let $(A, B)$ be an E.I. pair of sets which is separated by $F(x)$ in $(T)$. Thus $A \subseteq F_{T}$ and $B \subseteq F_{R}$. Since $(A, B)$ is E.I. then obviously the larger pair $\left(F_{T}, F_{R}\right)$ is E.I. And since $(T)$ is axiomatizable then $F_{T}$ and $F_{R}$ are both r.e. sets. And of course, $F$ exactly separates $\left(F_{T}, F_{R}\right)$ in $(T)$. So $\left(F_{T}, F_{R}\right)$ is an E.I. pair of r.e. sets which is exactly separable in $(T)$.

From Theorem 2 and Lemma 2 we now have

THEOREM 3. If $(T)$ is a consistent axiomatizable theory in which all recursive functions are definable and in which some E.I. pair of sets is separable, then $(T)$ is an exact Rosser theory.

${ }^{3}$ Or even every $1-1$ recursive function of one argument.

4 That is, we consider the Kleene predicate $T_{1}(z, x, y)$ (cf. [6, p. 281]) and define $\omega_{i}$ to be the set of all numbers $n$ satisfying the condition: $(\exists y) T_{1}(i, n, y)$. 
Our next theorem is an immediate consequence of Theorem 3 and the well known fact that there exists an E.I. pair of r.e. sets.

THEOREM 4. Every consistent axiomatizable Rosser theory in which all recursive functions are definable is an exact Rosser theory.

5. Applications. We now consider any consistent extension $(T)$ of Robinson's system $R$-or, in fact, any consistent theory $(T)$ in which all recursive functions are definable $e^{5}$ and in which there is a binary formula $x \leqq y$ with the properties:

(i) For all $n: \vdash x \leqq \bar{n} \supset(x=\overline{0} \vee x=\overline{1} \vee \cdots \vee x=\bar{n})$,

(ii) For all $n: \vdash x \leqq \bar{n} \bigvee \bar{n} \leqq x$.

Any such theory $(T)$ is a Rosser theory. For let $A, B$ be disjoint r.e. sets; let $f(x), g(x)$ be recursive functions which respectively enumerate $A$ and $B$; let $f(x), g(x)$ be respectively defined in $(T)$ by $F(x, y), G(x, y)$. Then by an obvious generalization of the well known argument of Rosser, the pair $(A, B)$ is separated in $(T)$ by the formula:

$$
(\exists x)(F(x, y) \wedge(\forall z)(z \leqq x \supset \sim G(z, y))) .
$$

We thus have:

THEOREM 5. If $(T)$ is any consistent axiomatizable extension of $R$ (or if $(T)$ is any axiomatizable theory obeying the above conditions) then $(T)$ is an exact Rosser theory-i.e. for every disjoint pair $(A, B)$ of r.e. sets there is a formula of $(T)$ which represents $A$ and whose negation represents $B$.

\section{REFERENCES}

1. A. Ehrenfeucht and S. Feferman, Representability of recursively enumerable sets in formal theories, Arch. Math. Logik Grundlagenforsch. vol. 5 (1960) pp. 37-41.

2. A. Tarski, A. Mostowski, and R. M. Robinson, Undecidable theories, Amsterdam, North-Holland Publishing Co., 1953.

3. J. Myhill, Creative Sets, Z. Math. Logik Grundlagen Math. vol. 1 (1955) pp. 97-108.

4. A. A. Muchnik, Isomorphism of systems of recursively enumerable sets with effective properties, Trudy Mosk ov. Mat. Obšc. vol. 7 (1958) pp. 407-412 (Russian).

5. R. M. Smullyan, Theory of formal systems, Doctoral Dissertation, Princeton, May 11, 1959. Also issued as a group report, \#54-5, Massachusetts Institute of Technology, Lincoln Laboratory. To be published as an Annals of Mathematics Study.

6. S. C. Kleene, Introduction to metamathematics, New York, Van Nostrand, 1952.

Princeton University

5 The definability of all recursive functions in every consistent extension of $R$ was established in [2]. Actually we only need the definability of all recursive functions of one argument. 